\title{
Muscarinic receptors promote pacemaker fate at the expense of secondary conduction system tissue in zebrafish
}

\author{
Martina S. Burczyk, ${ }^{1}$ Martin D. Burkhalter, ${ }^{1,2}$ Teresa Casar Tena, ${ }^{1}$ Laurel A. Grisanti, ${ }^{3}$ Michael Kauk, ${ }^{4}$ \\ Sabrina Matysik, ${ }^{1}$ Cornelia Donow, ${ }^{1}$ Monika Kustermann, ${ }^{1}$ Melanie Rothe, ${ }^{1}$ Yinghong Cui, ${ }^{5}$ \\ Farah Raad, ${ }^{6,7}$ Svenja Laue, ${ }^{8}$ Allessandra Moretti, ${ }^{8}$ Wolfram-H. Zimmermann, ${ }^{6,7}$ Jürgen Wess, ${ }^{5}$ \\ Michael Kühl, ${ }^{1}$ Carsten Hoffmann, ${ }^{4}$ Douglas G. Tilley, ${ }^{3}$ and Melanie Philipp ${ }^{1,2}$ \\ IInstitute of Biochemistry and Molecular Biology, Ulm University, Ulm, Germany. ${ }^{2}$ Department of Experimental and Clinical \\ Pharmacology and Pharmacogenomics, Division of Pharmacogenomics, University of Tuebingen, Tuebingen, Germany. \\ ${ }^{3}$ Center for Translational Medicine and Department of Pharmacology, Lewis Katz School of Medicine, Temple University, \\ Philadelphia, Pennsylvania, USA. ${ }^{4}$ Institute for Molecular Cell Biology, University Hospital Jena, Friedrich-Schiller University \\ of Jena, Jena, Germany. ${ }^{5}$ Molecular Signaling Section, Laboratory of Bioorganic Chemistry, National Institute of Diabetes \\ and Digestive and Kidney Diseases, National Institutes of Health, Bethesda, Maryland, USA. ${ }^{6}$ Institute of Pharmacology \\ and Toxicology, University Medical Center Göttingen, Göttingen, Germany. ${ }^{7}$ German Centre for Cardiovascular Research \\ (DZHK), partner site Göttingen, Germany. ${ }^{8}$ Medical Department - Molecular Cardiology, Technical University Munich, \\ Munich, Germany.
}

Deterioration or inborn malformations of the cardiac conduction system (CCS) interfere with proper impulse propagation in the heart and may lead to sudden cardiac death or heart failure. Patients afflicted with arrhythmia depend on antiarrhythmic medication or invasive therapy, such as pacemaker implantation. An ideal way to treat these patients would be CCS tissue restoration. This, however, requires precise knowledge regarding the molecular mechanisms underlying CCS development. Here, we aimed to identify regulators of CCS development. We performed a compound screen in zebrafish embryos and identified tolterodine, a muscarinic receptor antagonist, as a modifier of CCS development. Tolterodine provoked a lower heart rate, pericardiac edema, and arrhythmia. Blockade of muscarinic M3, but not M2, receptors induced transcriptional changes leading to amplification of sinoatrial cells and loss of atrioventricular identity. Transcriptome data from an engineered human heart muscle model provided additional evidence for the contribution of muscarinic M3 receptors during cardiac progenitor specification and differentiation. Taken together, we found that muscarinic M3 receptors control the CCS already before the heart becomes innervated. Our data indicate that muscarinic receptors maintain a delicate balance between the developing sinoatrial node and the atrioventricular canal, which is probably required to prevent the development of arrhythmia.

Conflict of interest: The lab of MP has received a research grant from the Boehringer Ingelheim Ulm University BioCenter, which is a research program that is cofunded by the Ministry of Research of the State of Baden-Wuerttemberg, Boehringer Ingelheim, and the Medical School at Ulm University.

Copyright: () 2019, American Society for Clinical Investigation.

Submitted: May 1, 2018

Accepted: August 27, 2019

Published: October 3, 2019

Reference information: /CI Insight. 2019:4(20):e121971.

https://doi.org/10.1172/jci.

insight.121971.

\section{Introduction}

The cardiac conduction system (CCS) is an integral component of the heart that initiates and maintains a regular heartbeat. Irregular development of CCS cells results in improper propagation of electrical excitation signals and consequently arrhythmia, which can lead to severe contractile dysfunction of the heart. Arrhythmia requires medical attention, such as symptomatic treatment with antiarrhythmic drugs and implantation of defibrillation or pacemaker devices (1). The best option to treat arrhythmia, however, would be to generate new functional pacemaker cells from existing cardiomyocytes at the proper anatomical location within the patient's heart. Such gene therapeutic strategies aiming at the reprogramming of existing cardiomyocytes to functional sinoatrial pacemaker cells will benefit from a detailed understanding of gene networks that guide embryonic conduction system development; in this context, it is notable that attempts to reestablish CCSs by reprogramming have recently been reported in pigs, with encouraging results (2-5). Pigs share a tremendous anatomical similarity with humans and are highly susceptible to arrhythmia. Rodents exhibit a very high heart rate, and despite having similar conduction system anatomy, 
there are important differences as to arrhythmia inducibility and sustainability in rodents versus humans. For screening applications, rodents or pigs are not well suited because of low throughput and high costs. Cell culture systems, on the other hand, are powerful systems for screening but lack the heart's anatomy, which is particularly important for CCS function. Thus, smaller lab animals, particularly aquatic models such as zebrafish, have been introduced. Zebrafish, despite having only a simple 2-chambered heart, have become a highly valued model for the analysis of both cardiac development as well as heart function (6). Similar to mammalian models, the zebrafish CCS is composed of a 2-tiered hierarchy of cardiac regions, the sinoatrial node (SAN) and the atrioventricular canal (AVC) (7). The SAN of zebrafish embryos develops at the interface of venous entry and atrium, while the second part of the conduction system resides between the atrium and the single ventricle. As in other models, SAN development relies on a gene regulatory network including Islet1 (Is11), Shox2, and T box factors $(8,9)$. How the conductive tissue of the AVC emerges, however, is less clear, although several marker genes are available to analyze the state of the developing AVC (10).

With the aim to find additional ways to decipher and to manipulate CCS development, we have performed a small chemical compound screen in zebrafish embryos and looked for drugs able to induce arrhythmia. Such screens have previously been proved instrumental in the identification of new applications for old, established drugs $(11,12)$. We identified tolterodine, an antimuscarinic agent clinically used for the treatment of overactive bladder, as a pacemaker-modifying agent in zebrafish embryos and conducted further detailed analyses in mice and human cells. Our data suggest that at least in zebrafish, tolterodine promotes SAN fate at the expense of AVC development before the heart is innervated.

\section{Results}

Compound screen for arrhythmogenic drugs. Cardiogenesis in zebrafish largely resembles mammalian heart development. Cells giving rise to cardiac progenitor cells (CPCs) can be traced as early as 5 hours after fertilization (hpf), which is even before gastrulation, during which cells gradually pause in proliferation and exhibit migratory behavior. At the end of gastrulation (10 hpf), organogenesis starts and the first CPCs are specified (12 hpf). CPCs are arranged in heart fields bilaterally to the midline of the embryo and undergo further differentiation into atrial, ventricular, and endocardial lineages (around $15 \mathrm{hpf}$ ) before they migrate to the midline and fuse to a ring-like structure $(19 \mathrm{hpf})$. A simple heart tube extends from this ring of cells and soon after starts contracting ( $24 \mathrm{hpf}$ ), looping (36 hpf), and turning into a 2-chambered heart with a functional CCS (48 hpf) (13). To screen for novel regulators of the CCS in zebrafish embryos, we used a compound library of 90 FDA-approved, pharmacologically active drugs with known targets. To prevent organ defects arising from interfering with cell movements during gastrulation, zebrafish embryos were treated from tailbud stage (10 hpf) until we performed analyses at $48 \mathrm{hpf}$. In an initial screen using 5 embryos per compound, 8 compounds produced arrhythmia in zebrafish, which was never observed in vehicle-treated embryos. After rescreening these compounds in larger cohorts, we reproduced the arrhythmogenic potential for all 8 agents; however, only 2 compounds, PF-4708671 and tolterodine, resulted in arrhythmia statistically different from DMSO (Figure 1A and Supplemental Figure 1; supplemental material available online with this article; https://doi.org/10.1172/jci.insight.121971DS1). In addition, we assumed that cardiac performance is impaired when arrhythmia developed and assessed the occurrence of pericardiac and inflow tract edema. PF-4708671, an inhibitor of S6K1, and tolterodine, a muscarinic receptor antagonist, caused edema (Figure 1B). We also observed that tolterodine treatment resulted in highly variable heart rates, probably because of the occurrence of arrhythmias (Figure 1C). We therefore chose tolterodine for further analysis.

Tolterodine causes arrhythmia concentration dependently. Next, we performed tolterodine dose-response experiments to revalidate the effects described above. Again, embryos were treated from tailbud stage until $48 \mathrm{hpf}$ and were subsequently scored for atrial arrhythmia, for conduction defects between the atrium and the ventricle, and for overall cardiac impairment. Increasing concentrations of tolterodine caused atrial arrhythmia (Figure 2A) and atrioventricular blocks (AV-blocks) (Figure 2B) with silent ventricles from $5 \mu \mathrm{M}$ onward. Similarly, pericardiac and inflow tract edema also developed concentration dependently (Figure 2, C and D), suggesting that the observed defect is unlikely to be due to nonspecific toxic side effects of tolterodine.

Combined M2/M3 abrogation affects cardiac performance in other species. To exclude the possibility that the observed phenotype is zebrafish specific, we investigated the impact of muscarinic receptor loss of function 

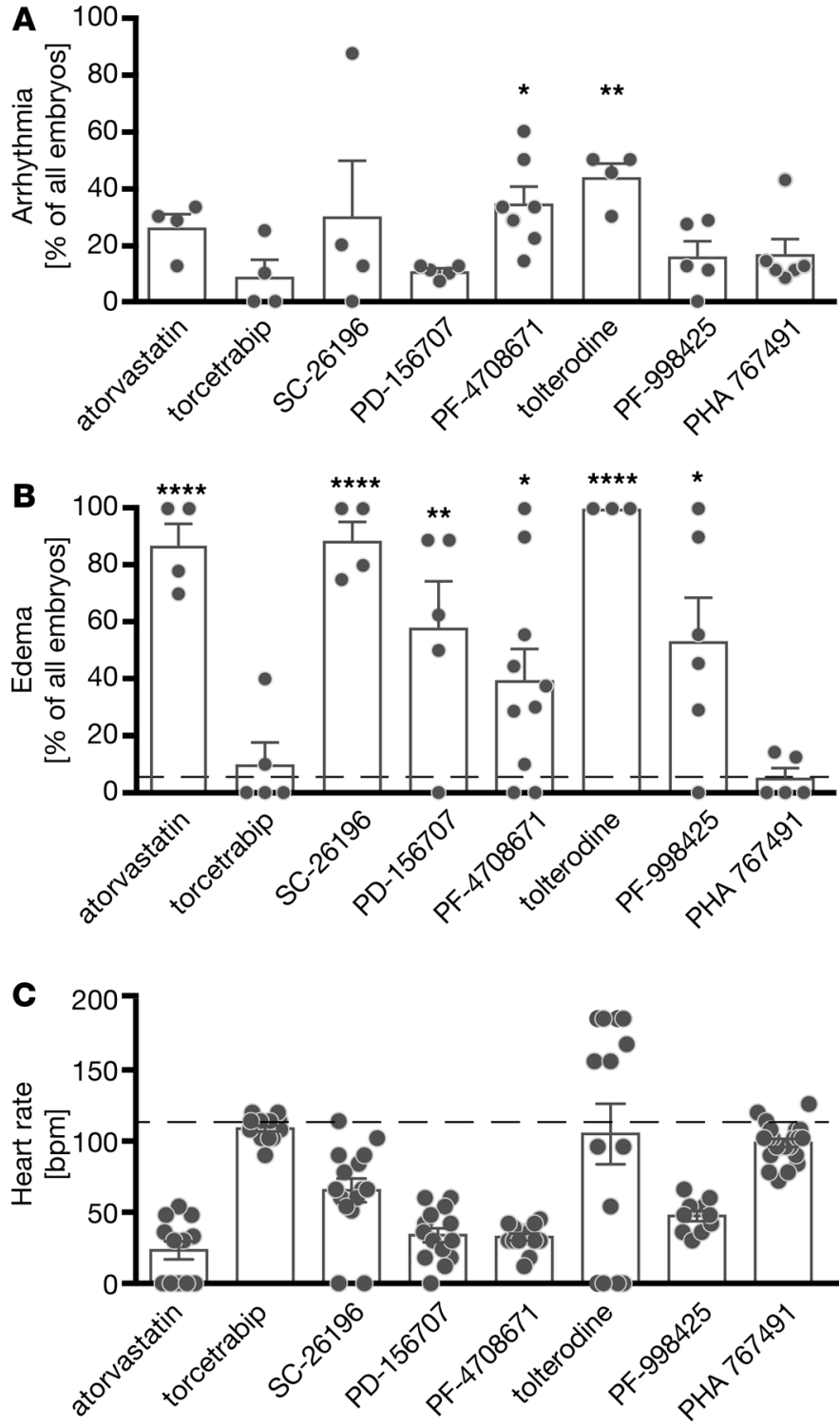

Figure 1. Compound screen reveals 9 drugs causing cardiac arrhythmia in zebrafish. Zebrafish embryos were cultured in $50 \mu \mathrm{M}$ compound in egg water or in egg water containing $1 \%$ DMSO from tailbud stage on. At the $48 \mathrm{hpf}$ stage, embryos were individually and manually assessed for arrhythmia (A), formation of pericardiac or inflow tract edema (B), and atrial heart rates (C). (A) Eight out of the 90 drugs of the LOPAC Pfizer library produced arrhythmia in zebrafish embryos. DMSO-treated embryos never showed arrhythmia. $n$ = 4-7 treatments with 31-56 embryos. (B) Seven drugs impaired overall heart function, resulting in edema formation. Dashed line indicates the percentage of embryos developing edema under DMSO treatment. $n=4-7$ treatments with 25-83 embryos. (C) Tolterodine-treated embryos showed the highest variability in heart rate (shown as bpm) compared with DMSO-treated embryos (dashed line). Circles display heart rates of individual embryos. $n$ $=10-158$ embryos. Bar graphs display mean \pm SEM, with circles indicating individual experiments. (A and B) Data analyzed by 1 -way ANOVA, with ${ }^{*} P<$ $0.05,{ }^{*} P<0.01$, and ${ }^{* * *} P<0.0001$.

in 2 additional species. First, we turned to Xenopus laevis embryos. Treatment with tolterodine from stage 12.5 until 42 produced pericardiac edema, arrhythmia, and bradycardia, as observed in zebrafish (Figure 3). In addition, we analyzed double-KO mice lacking both M2 and M3 receptors (Figure 4). Tolterodine blocks all 5 muscarinic receptor subtypes (M1-M5) with similar potency (14). Cardiac tissue almost exclusively expresses M2 and M3 muscarinic receptors (15), and mice lacking either M2 or M3 receptors have been generated in the past $(16,17)$. None of the $2 \mathrm{KO}$ strains, however, showed changes in cardiac performance under unchallenged conditions $(18,19)$, while double-mutant mice showed striking phenotypes. From double-homozygous matings (Chrm2 $2^{--} \mathrm{Chrm}^{3^{--}} \times \mathrm{Chrm}^{-/-} \mathrm{Chrm}^{-/-}$), 85 out of 372 mice in total were found dead after weaning (corresponding to 22\%), and 70 out of 442 mice died before weaning (16\%). Moreover, a substantial number of animals displayed physical malformations, such as hydrocephalus (11 of 372), and could not be used for experiments. In the surviving double-KO mice, we assessed cardiac parameters. Although we did not observe an overt difference in rhythm or the heart rate (Figure 4, A and B), which may be attributed to a selection bias introduced by the high pre- and postweaning mortality, there were significant functional differences. We observed an increased heart to body weight ratio, which may at least partially be due to the overall smaller size of the mice (Figure 4C). M2/M3 double-KO mice displayed an overall decreased contractility (Figure 4, D and $\mathrm{E}$ ), which was accompanied by dilatation of the left ventricle (Figure 4, F and G). However, because of the fragile nature of the mice, we could not assess this in greater detail. In summary, these data suggest that the role of muscarinic receptors in the control of heart function is conserved in vertebrates. 

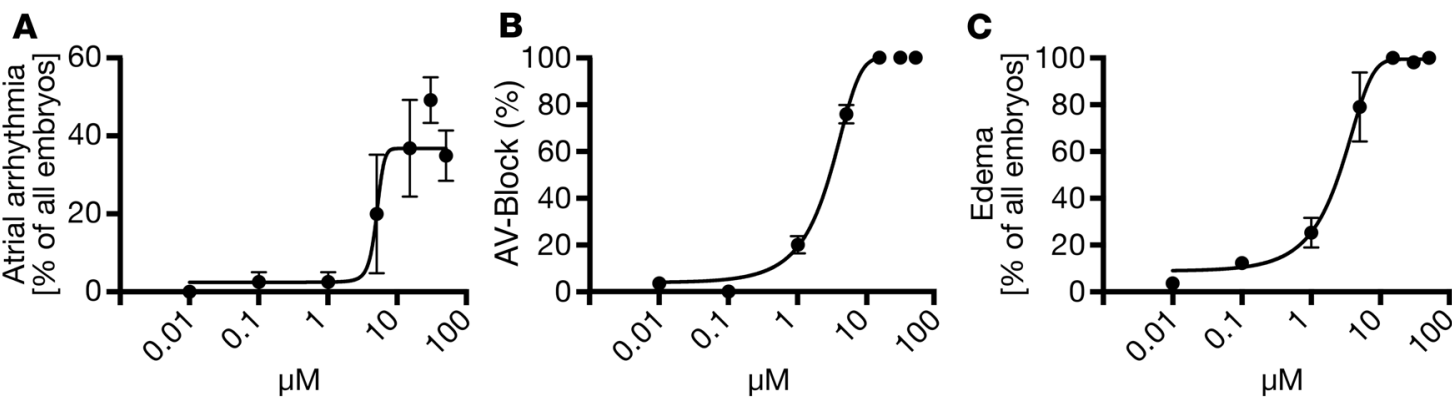

D
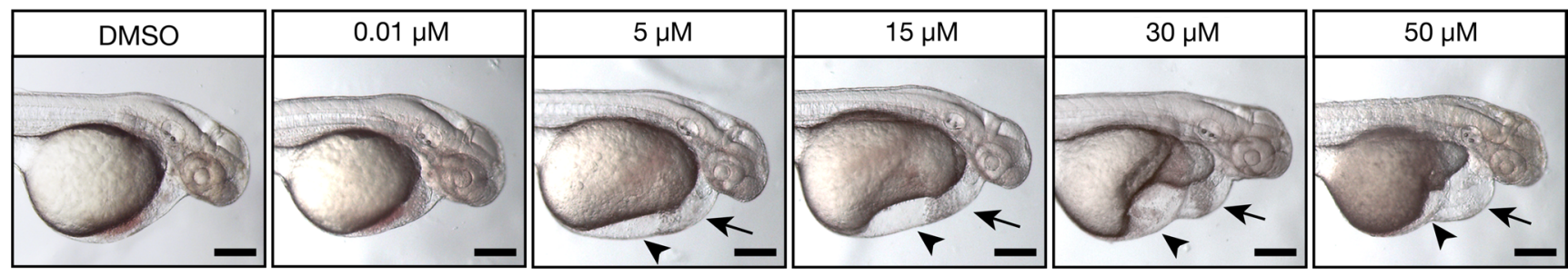

Figure 2. Cardiac performance and conduction system function are compromised by increasing concentrations of tolterodine. (A) Zebrafish embryos were treated from tailbud stage on with increasing concentrations of tolterodine. At $48 \mathrm{hpf}$, embryos were assessed for atrial arrhythmia. $n=3-11$ experiments with 74-137 embryos. (B) Tolterodine-treated embryos developed atrioventricular conduction blocks (AV-blocks). A value of $50 \%$ corresponds to a 2:1 AV-block with the atrium beating twice as fast as the ventricle; $100 \%$ AV-block indicates a silent ventricle. $n=45-56$ embryos. (C) Zebrafish were also assessed for edema formation as a general readout for impaired cardiac function. $n=4$ experiments with $73-85$ embryos. (D) Live images of 48 hpf embryos after treatment with increasing concentrations of tolterodine. Arrows indicate pericardiac edema and arrowheads inflow tract edema. Scale bar: $200 \mu \mathrm{m}$. All graphs display mean $\pm \mathrm{SEM}$.

Tolterodine affects heart function primarily via M3 muscarinic receptors. In principle, zebrafish have all 5 muscarinic receptors (Chrm1-5), of which M2, M3, and M5 exist as duplicated genes on different chromosomes (Chrm2a/chromosome 4, Chrm2b/chromosome 25, Chrm3a/chromosome 17, Chrm3b/ chromosome 12, Chrm5a/chromosome 17, Chrm5b/chromosome 20). For the purpose of this study, we focused on the zebrafish orthologs of the human M2 and M3 receptors. M2a encoded by chrm2a has the highest sequence similarity to human M2, while M3a encoded by chrm3a shows the highest sequence homology with human M3 (Table 1 and Supplemental Figures 2 and 3). To demonstrate that M2a and $\mathrm{M} 3 \mathrm{a}$ are functional muscarinic receptors, we performed a modified bioluminescence resonance energy transfer (BRET) assay for $\beta$-arrestin 2 recruitment (20). Here, HEK293T cells were cotransfected with Halo-tagged $\beta$-arrestin 2, G protein-coupled receptor kinase 2 (GRK2), and a fusion of either M2a or M3a with a C-terminal nanoluciferase. When both zebrafish receptors are functional, agonist-induced receptor activation should lead to the recruitment of $\beta$-arrestin 2 and a concentration-dependent bioluminescence signal. In fact, both acetylcholine as well as the synthetic ligand carbachol led to efficient recruitment of $\beta$-arrestin 2, suggesting that both ligands act as efficacious agonists at M2a and M3a (Supplemental Figure $4, \mathrm{~A}$ and B). Moreover, tolterodine efficiently abrogated $\beta$-arrestin 2 recruitment by either M2a or M3a upon agonist stimulation (Supplemental Figure 4, C and D). We therefore conclude that M2a and M3a are functional orthologs of the human M2 and M3 receptors, respectively.

We next studied whether the tolterodine-induced heart phenotype in zebrafish originated from blocking either muscarinic receptors resembling M2 or M3. None of the M2 receptor-preferring antagonists tested (himbacine, AF-DX 116) resulted in atrial arrhythmia, edema, or the expected increase in heart rate in $48 \mathrm{hpf}$ embryos (Figure 5), although AF-DX 116 proved to have antagonistic function at zebrafish M2a (Supplemental Figure 4E). When we treated from $48 \mathrm{hpf}$ on, however, and assessed the heart rate at $72 \mathrm{hpf}$, we observed tachycardia upon M2 receptor inhibition $\left(\mathrm{DMSO}_{48-72 \mathrm{hpf}}: 157.6 \pm 1.759 \mathrm{bpm}\right.$, and $n=87$ embryos; $50 \mu \mathrm{M}$ AF-DX 116 ${ }_{48-72 \mathrm{hpf}}: 170.3 \pm 3.025$ bpm, 83 embryos; $P<0.0001$, Mann-Whitney $U$ test), indicating that $\mathrm{M} 2$ receptors are functional at a later developmental stage. Similarly, knockdown of the M2a receptor, a homolog in zebrafish, by morpholino oligonucleotide (MO) injection produced tachycardia at $72 \mathrm{hpf}$ without affecting other cardiac parameters (Supplemental Figure 5). This observation of tachycardia at later stages is consistent with an earlier study carried out in zebrafish (21). We noted that zebrafish hearts were not yet innervated $48 \mathrm{hpf}$ (Supplemental Figure 6), suggesting that the 


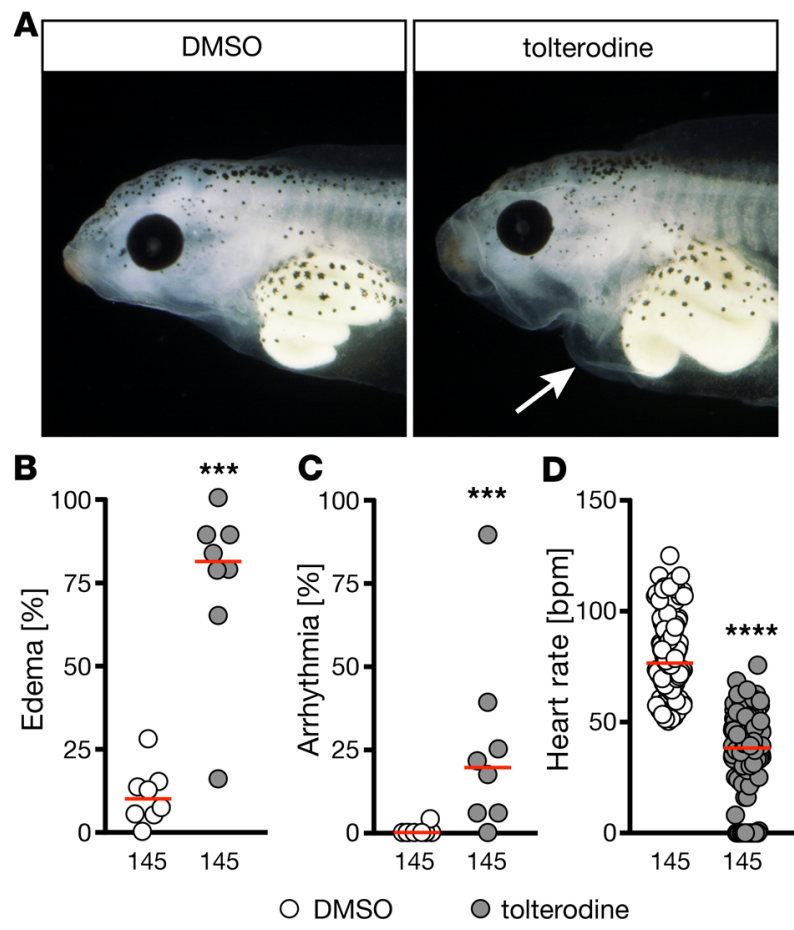

Figure 3. Tolterodine affects heart function in Xenopus in a similar fashion as in zebrafish. Xenopus embryos were treated from stage 12.5 until 42 in frog water and analyzed by hand. In B-D numbers of animals used are indicated below the graphs. (A) Live images of stage 42 Xenopus embryos treated with vehicle ( $1 \% \mathrm{DMSO})$ or $50 \mu \mathrm{M}$ tolterodine. Arrow indicates pericardiac effusion. (B) Tolterodine treatment results in pericardiac edema in Xenopus embryos. $n=8$ experiments. ${ }^{* * *} P$ $=0.0002$, 2-tailed Mann-Whitney $U$ test. (C) A significant number of Xenopus embryos developed arrhythmia upon tolterodine treatment. $n=8$ experiments. ${ }^{* *} P=0.0007$, 2-tailed Mann-Whitney $U$ test. (D) Tolterodine induced bradycardia in Xenopus embryos. $n=8$ experiments. ${ }^{* * *} P<0.0001,2$-tailed Mann-Whitney $U$ test. Graphs show mean of individual experiments ( $B$ and $\mathbf{C}$ ) or individual embryos (D). Red line, median.

parasympathetic system, which mediates its cardiac actions via stimulation of M2 receptors, becomes active only after $48 \mathrm{hpf}$.

We then carried out concentration response experiments using 2 muscarinic antagonists that preferentially block M3 receptors, solifenacin and zamifenacin $(14,22)$. Zamifenacin was also tested in our BRET assay, where it concentration-dependently abrogated receptor-dependent $\beta$-arrestin 2 recruitment (Supplemental Figure 4F). In zebrafish, increasing concentrations of both compounds caused arrhythmia, AV-blocks, and edema. Zamifenacin was even more potent than tolterodine and caused silent hearts from $10 \mu \mathrm{M}$ on (Figure 6). To further corroborate the involvement of M3 receptors, we performed MO injections to knock down M3a. Depletion of M3a via MO injection at the 1-cell stage proved detrimental for embryos and required very careful dosing of the MO to allow for survival up to 3 days after fertilization. Embryos injected with an intermediate dose of M3a MO (Supplemental Figure 7A), however, could be followed until $72 \mathrm{hpf}$ and developed pericardiac edema and arrhythmia (Supplemental Figure 7). These data suggest that tolterodine modulates early heart function via an M3-like receptor.

Tolterodine treatment affects heart morphology. During development heart function is tightly coupled to cardiac morphology (23). Thus, the tolterodine-induced conduction defects may potentially arise from a malformation of the developing heart. In fact, zebrafish receiving tolterodine developed dose-dependently elongated AVCs and smaller, deformed ventricles (Figure 7A). This phenotype was not associated with a change in cardiomyocyte number (Figure 7B) or increased apoptosis (Figure 7C). Additionally, chamber specification into atrium and ventricle remained unaffected (Figure 7D).

Tolterodine drives pacemaker expansion at the expense of AVC conduction tissue. To better understand the cause of tolterodine-induced arrhythmia, we analyzed the 2 main components of the CCS in zebrafish (SAN and AVC). First we assessed expression of anf, which is normally excluded from noncontractile myocardium (24). Consistent with our observation of atrioventricular conduction defects, we detected anf transcripts throughout the entire heart when embryos were treated with tolterodine (Figure 8A) or injected with the M3a MO (Supplemental Figure 8). Control embryos, on the other hand, lacked anf expression in the AVC (Figure 8A). Furthermore, notch1b, a marker for conductive cells of the AVC, was absent after tolterodine treatment (Figure $8 \mathrm{~B}$ ). Other markers of the AVC were also deregulated, for example, cspg2a, which was dispersed throughout the whole heart (Supplemental Figure 9A), and tbx2b, which was no longer enriched in the AVC of tolterodine-treated embryos (Supplemental Figure 9B). Intriguingly, genes indicative of pacemaker cells were expressed at higher levels in tolterodine-treated embryos. All 4 genes tested, fgf13a (Figure 8C), tbx18 (Figure 8D), isl1, and hon4 (Supplemental Figure 9, C and D), were 
A

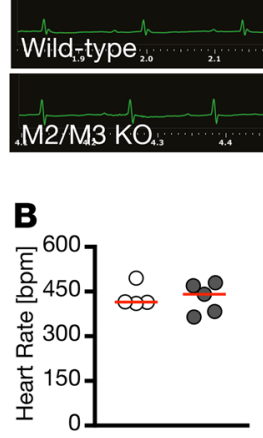

D

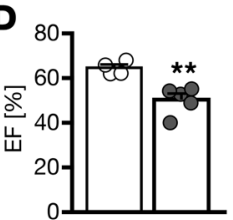

$\mathbf{F}$

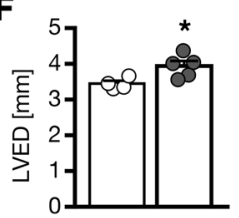

$\underbrace{1, \ldots \ldots \ldots, \ldots, 1,3}_{2,2}$ 2.3. ...........

O Wild-type

C

- M2/M3 KO

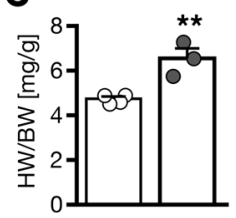

E

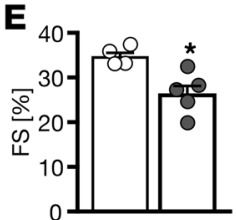

G

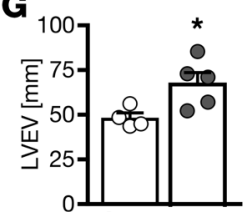

Figure 4. Mice lacking both M2 and M3 muscarinic receptors show impaired cardiac performance. (A) Representative echocardiograms of WT and M2/M3 double-KO mice reveal no abnormalities in cardiac rhythm. $n=4-5$ mice per genotype (5-6 months of age, mixed sex). (B) Heart rate was not altered in M2/M3 double-KO mice. $n=4-5$ mice per genotype. (C) $\mathrm{M} 2 / \mathrm{M} 3$ double-KO mice had a higher heart weight (HW) to body weight (BW) ratio. This was due to an overall reduced size of M2/M3 double-KO mice. ${ }^{* *} P=0.0057,2$-tailed $t$ test. $n=4-5$ mice per genotype. (D) M2/M3 double-KO mice had a reduced ejection fraction (EF). ${ }^{*} P=0.0040$, 2-tailed $t$ test. $n=4-5$ mice per genotype. (E) Fractional shortening (FS) was also reduced in $\mathrm{M} 2 / \mathrm{M} 3$ double-KO mice. ${ }^{*} P=0.0129$, 2-tailed $t$ test. $n=4-5$ mice per genotype. (F) At the same time, left ventricular end-diastolic diameter (LVED) was increased in M2/M3 double-KO mice. ${ }^{*} P=0.0272$, 2-tailed $t$ test. $n=$ 4-5 mice per genotype. (G) M2/M3 double-KO mice further displayed a larger left ventricular end-diastolic volume (LVEV). ${ }^{*} P=0.0303,2$-tailed $t$ test. $n=4-5$ mice per genotype. Bar graphs show mean \pm SEM and circles indicate individual mice.

either more abundant upon tolterodine treatment or appeared in a diffuse pattern in the atrium. Quantitative PCR (qPCR) studies with RNA from isolated hearts confirmed the in situ hybridization results: Tbx18, which was shown to induce pacemaker fate in some experimental systems (4), was upregulated in tolterodine hearts. Consistently, the pacemaker gene hcn 4 was increased, while connexin 43 (CX43) levels were decreased (Figure $8 \mathrm{E}$ ). These results suggest that tolterodine promotes sinoatrial development at the expense of the AVC in zebrafish.

Tolterodine CCS development at the level of CPCs. To study the effects of tolterodine in human heart development, we applied tolterodine to cardiac myocytes differentiated from human induced pluripotent stem cells (iPSCs). Three days of tolterodine treatment did not change the expression of $N k \times 2$ 5, which served as a marker of CPCs or immature cardiomyocytes. However, both Hcn4 and Shox2 expression levels were found to be elevated. Interestingly, this occurred similarly at the concentrations tested (5 and $50 \mu \mathrm{M}$ ) (Figure 9A). The presence of M3 receptors in the developing human heart was further confirmed by RNA-Seq in an organoid model of heart development (data not shown). Following a transient decrease in M3 receptors' transcript abundance during mesoderm specification, we found an upregulation during cardiac specification and maturation, with highest levels at the time point of heart muscle consolidation (days 22 and 29 after the start of directed self-assembly of undifferentiated pluripotent stem cells [HES2 line]). Consistent with this observation, chrm3a expression was detectable in zebrafish during CPC stages and increased during stages of CPC differentiation (Figure 9C). To evaluate the importance of M3 receptors during CPC stages, we performed multiple

Table 1. Sequence identity and similarity (in parentheses) for M2 and M3 receptors

\begin{tabular}{|c|c|c|c|c|}
\hline & DrM2a & DrM2b & DrM3a & DrM3b \\
\hline HsM2 & $69.5 \%(81.9 \%)$ & $58.3 \%(77.3 \%)$ & $43.1 \%(68.4 \%)$ & $45.4 \%(70.7 \%)$ \\
\hline HsM3 & $43.8 \%(70.9 \%)$ & $45.9 \%(72.1 \%)$ & $63.0 \%$ (83.0\%) & $56.1 \%(72.6 \%)$ \\
\hline
\end{tabular}



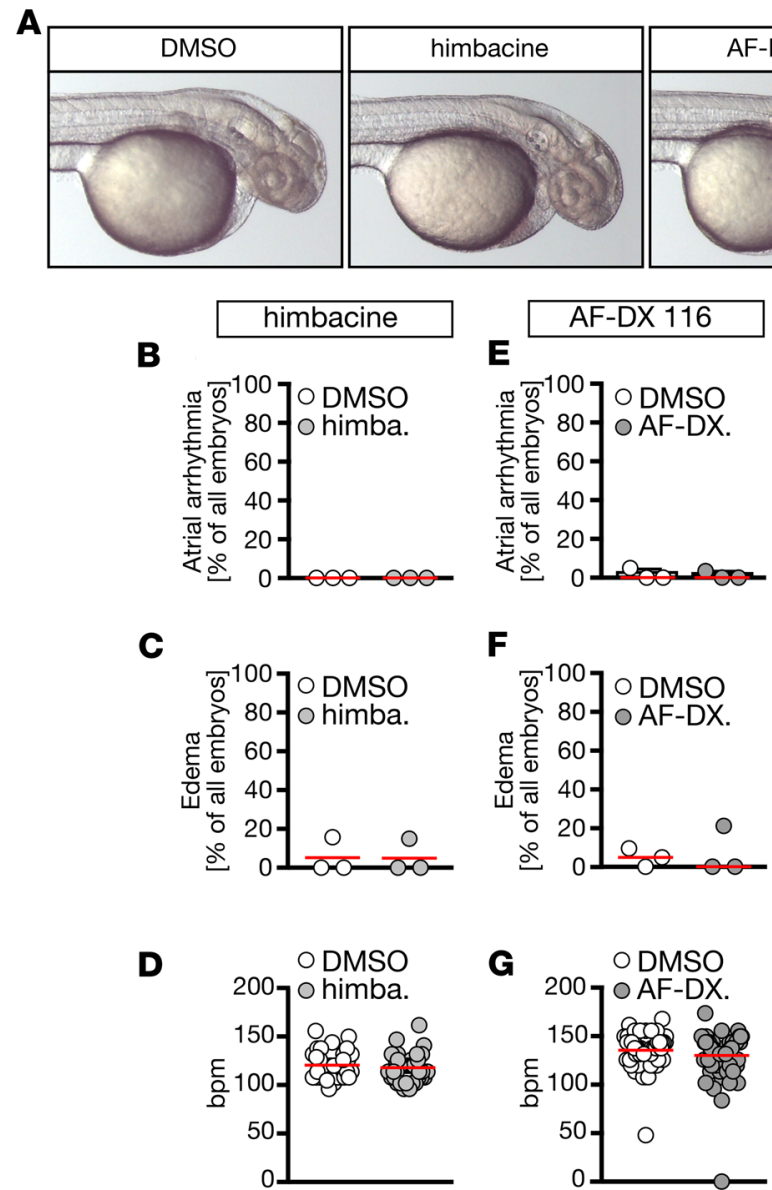

Figure 5. Zebrafish treatment with antimuscarinic drugs favoring M2 over M3 receptors does not phenocopy the effects seen with tolterodine. (A) Live images of $48 \mathrm{hpf}$ embryos after treatment with vehicle (DMSO) or $50 \mu \mathrm{M}$ himbacine (himba.) or AF-DX 116 (AF-DX.). Scale bar: $200 \mu \mathrm{m}$. (B) Himbacine treatment did not result in arrhythmia. $n$ = 3 experiments with 63-65 embryos. (C) Himbacine did not produce edema. $n=3$ experiments with 63-65 embryos. (D) The heart rate was not changed upon himbacine treatment. $n=63-65$ embryos. (E) AF-DX116 treatment did not promote arrhythmia development. $n=3$ experiments with 62-67 embryos. (F) Edema did not develop upon AF-DX 116 treatment. $n=3$ experiments with 62-67 embryos. (C) Heart rates were not changed when treated with AF-DX116. $n=$ 62-67 embryos. Circles indicate individual experiments (B, C, E, and $\mathbf{F}$ ) or embryos ( $\mathbf{D}$ and $\mathbf{C}$ ); median is shown with red line. All data analyzed by 2-tailed Mann-Whitney $U$ tests.

time course experiments, in which zebrafish embryos were treated with tolterodine for various times between tailbud stage and $48 \mathrm{hpf}$. We observed that tolterodine treatment during cardiac specification and differentiation stages was sufficient to induce AVC elongation in more than $50 \%$ of all embryos (Figure 9D). Moreover, tolterodine exposure from tailbud to 20 ss robustly induced anf expression in the AVC and more abundant and diffuse expression of isl1 (Figure 9, E and F), indicating that M3 receptor signaling limits pacemaker potential during development.

\section{Discussion}

In the current study, we have used a small compound screen in zebrafish embryos to gain potentially novel insights into the molecular mechanism underlying CCS development. We found that tolterodine, a muscarinic receptor antagonist, concentration-dependently affects cardiac performance and rhythmicity. Tolterodine treatment alters the shape of the heart without changing the overall number of cardiomyocytes or affecting chamber specification. Closer analysis revealed that tolterodine, most likely via inhibition of M3 muscarinic receptors, evokes transcriptional changes indicative of pacemaker fates at the expense of the AVC.

We found that M3 muscarinic receptors are expressed and tightly regulated during cardiac progenitor stages. Consistent with this observation, blockade of muscarinic receptors during cardiac progenitor 
A
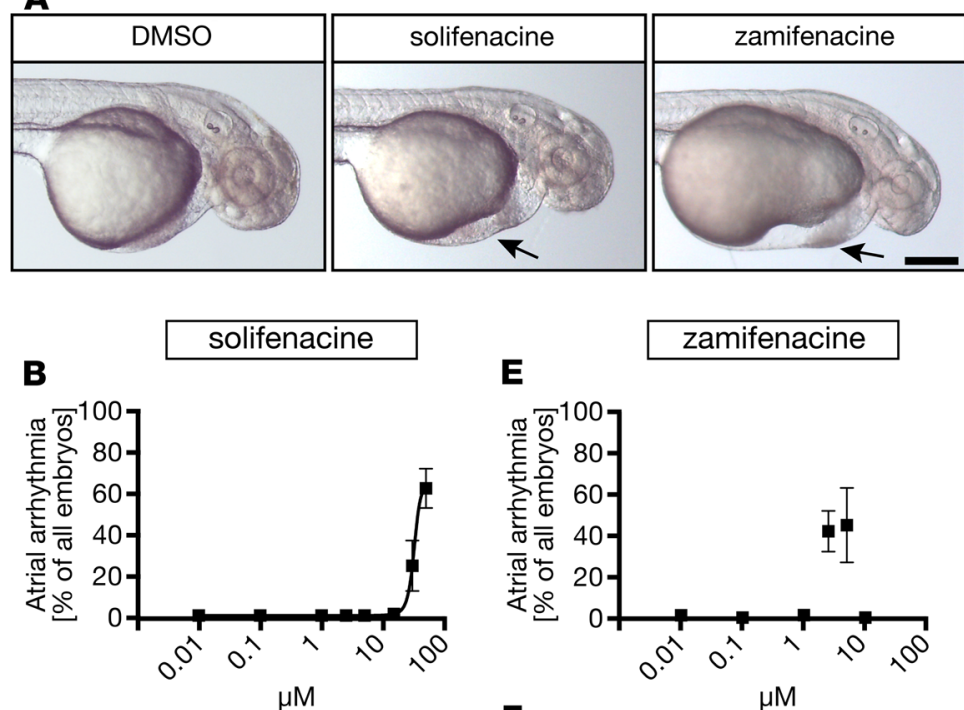

C

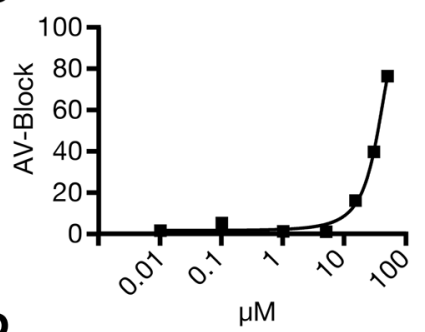

D

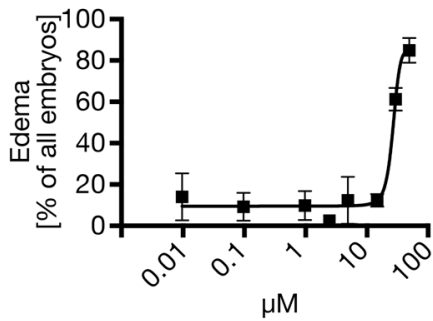

\section{E}

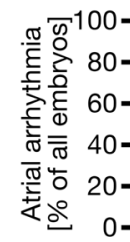

F

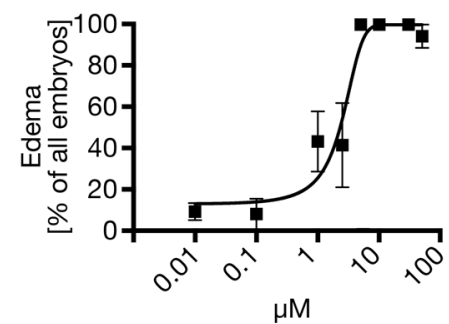$$
\text { G }
$$

Figure 6. Antimuscarinic compounds favoring $\mathrm{M} 3$ over $\mathrm{M} 2$ receptors phenocopy tolterodine treatment in zebrafish embryos. (A) Live images of 48 hpf embryos after treatment with vehicle (DMSO) or $50 \mu \mathrm{M}$ solifenacin or zamifenacin. Arrow indicates the edema. Scale bar: $200 \mu \mathrm{m}$. (B) Solifenacin treatment caused dose-dependent arrhythmia. $n$ = 3-8 experiments with 85-165 embryos. (C) Zebrafish treated with increasing concentrations of solifenacin developed AV-blocks. $n=3-8$ experiments with 85-165 embryos. (D) Solifenacin caused formation of edema with increasing concentrations. $n=3-8$ experiments with 85-165 embryos. (E) Zebrafish embryos treated with zamifenacin developed arrhythmia at low micromolar concentrations. At higher concentrations, both chambers became silent. $n=3-6$ experiments with 59-144 embryos. (F) Zamifenacin treatment provoked AV-blocks. $n=3-6$ experiments with 59-144 embryos. (G) Edema formation occurred in a dose-dependent manner upon zamifenacin treatment. $n=3-6$ experiments with 59-103 embryos. Graphs display mean \pm SEM.

stages was sufficient to produce an elongated AVC and aberrant marker gene expression. Cardiac progenitors are currently divided into 2 populations, the first and second heart field (25). A subset of second heart field cells is thought to give rise to the venous pole of the heart and as such most likely also to the SAN. Our results suggest that muscarinic receptors may constrain second heart field cells and limit the number of cells differentiating into pacemakers. On the other hand, it is also possible that there exists a lineage of progenitor cells, which are distinct from precursors giving rise to contractile myocardial cells or originate from them. In fact, a third heart field giving rise to pacemaker cells has been proposed previously $(26,27)$. Other theories consider conduction system cells as not fully differentiated, noncontractile cardiomyocytes (28). This would imply that inhibition of muscarinic receptors prevents full differentiation of cardiomyocytes or mediates partial dedifferentiation of mature cardiomyocytes. Another possibility is a direct antagonistic feedback loop between the SAN and AVC, which is balanced by the muscarinic system. Both the sinoatrial and a subset of the AVC cells share a common lineage of Islet1-positive cells (28). In chicken embryos, Islet1 forms a gradient from the sinus venosus to the developing AVC, establishing crosstalk between the 2 tiers of the CCS (28). Although we did not observe a gradient of Islet1 expression, we did detect islet1-positive cells distributed throughout the lower half of the atrium.

The adult CCS is controlled by the autonomous nervous system, which regulates the activity of cardiac muscarinic receptors. The embryonic CCS similarly serves as the orchestrator of steady contractions and, according to our data, depends on muscarinic receptor signaling. Muscarinic inhibition, however, confines the developing CCS already before the heart is innervated. At the stage we observed 

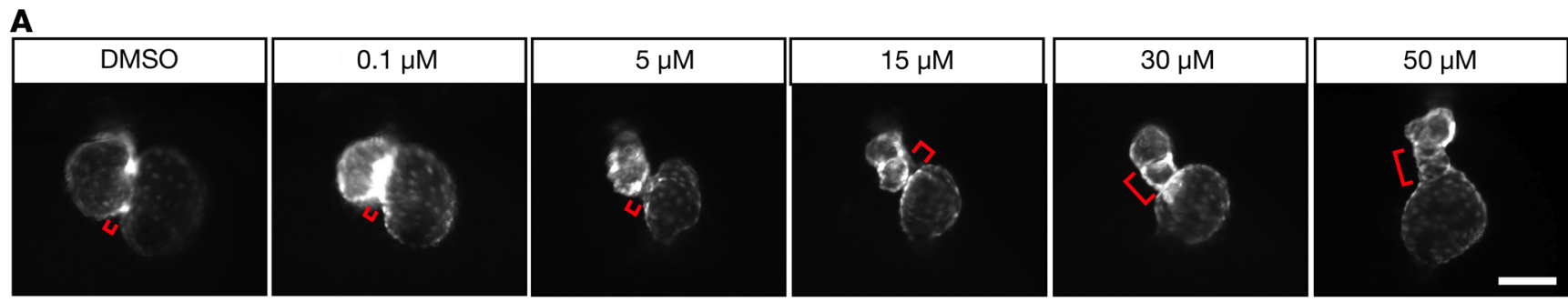

$\mathbf{8}$
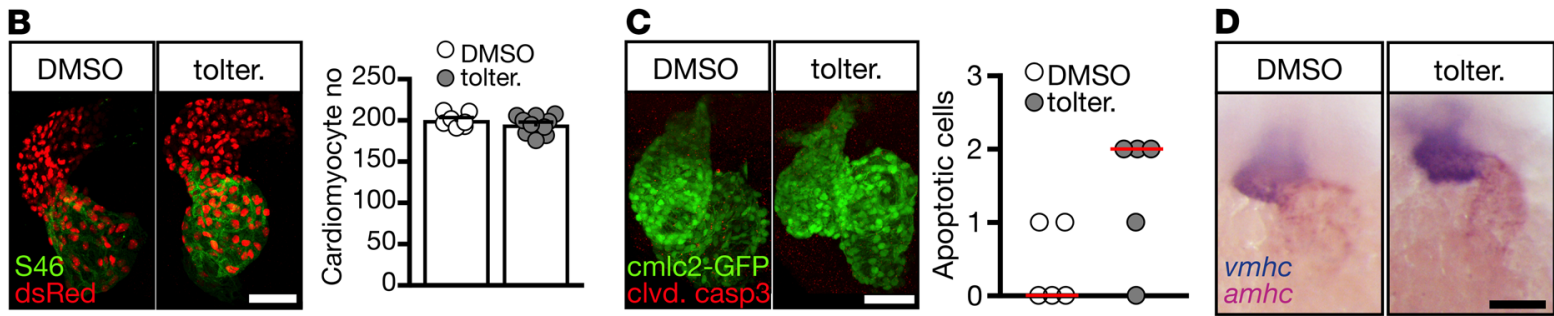

Figure 7. Tolterodine treatment causes elongation of the AVC. (A) Whole-mount live images of fluorescent zebrafish hearts [Tg(myI7:EGFP)] at $48 \mathrm{hpf}$. The AVC (indicated by a red bracket) is elongated upon tolterodine treatment. (B) Cardiomyocyte number remained unaltered in tolterodine-treated zebrafish. Images show confocal stacks of $\mathrm{Tg}(-5.1 \mathrm{my} / 7$ :DsRed2-NLS) zebrafish. DsRed-expressing nuclei of cardiomyocytes were enhanced by staining with an anti-DsRed antibody. Atria were visualized by staining of atrial myosin (S46). Graph shows individual embryos as circles and mean \pm SEM. $n=7-10$ embryos. (C) Tolterodine did not induce apoptosis in cardiomyocytes as shown by the lack of cleaved caspase-3positive cardiomyocytes. Images show confocal stacks of $T g$ (myl7:EGFP) hearts. Circles show individual embryos. $P=0.1429,2$-tailed Mann-Whitney $U$ test. $n=5$ embryos each. (D) Chamber specification was not affected by tolterodine. Atrial (amhc) and ventricular ( $v m h c$ ) myosin visualized by double in situ hybridization. $n=3$ experiments with 34-50 embryos. Scale bars: $100 \mu \mathrm{m}$ (A), $50 \mu \mathrm{m}$ (B-D).

tolterodine-induced arrhythmia in zebrafish, we found axons only extending toward, but not touching, the sinus venosus. Similarly, in rodents cardiac ganglia form long after the new necessary structures of the conduction system have developed, and innervation does not start before E18.5. In fact, functional innervation of the SAN has been demonstrated postnatally only $(29,30)$. These observations are intriguing because they imply a heart-autonomic function of muscarinic receptors, which could modulate cardiac cell fates. We found that tolterodine induces expression of genes required for progenitor differentiation into pacemaker cells. One of these, $\mathrm{Tbx} 18$, has recently been reported to be sufficient to turn contractile cardiomyocytes into cells with pacemaker activity $(4,31)$. Moreover, gene transfer of Tbx18 successfully generated ectopic pacemakers in SAN-ablated pigs and restored rhythmic contractions (2). Myocardial Tbx8 overexpression alone, however, was not sufficient to induce SAN fate in mice (32), suggesting that depending on the species or the experimental protocol used, additional factors may be needed to successfully turn contractile cells into pacemaker cells.

Taken together, our data suggest that muscarinic receptors, and here probably M3 receptors, not only control functionality of the mature CCS but also regulate the development of the embryonic CCS. In the future, it would be interesting to see whether targeted inhibition of muscarinic receptors could confer induction of new pacemaker cells in adult hearts and restore rhythmicity under conditions in which SAN functionality has been lost. Because muscarinic receptors are membrane proteins and can be readily blocked by orally available antimuscarinic agents, such an approach would be less invasive than gene transfer of Tbx18, especially because side effects of tolterodine treatment, such as dry mouth or constipation, are usually rather mild. Further studies are also needed to dissect the precise signaling cascade by which the M3 muscarinic receptor controls pacemaker gene expression in the embryo. For sure, however, muscarinic inhibition to ameliorate in adult arrhythmias, such as arrhythmogenic cardiomyopathy, which is characterized by life-threatening arrhythmia and sudden cardiac death as well as substantial myocardial remodeling (33), could represent an important line of future research.

\section{Methods}

Antibodies and reagents. All antibodies were obtained commercially: mouse anti-acetylated tubulin (MilliporeSigma, clone 6-11B-1, 1:1000), rabbit anti-cleaved caspase-3 (Cell Signaling Technology, catalog 9661S, 1:500), rabbit anti-DsRed (Clontech, catalog 632496, 1:500), and mouse anti-S46 (Developmental Studies Hybridoma Bank, 1:100). Secondary antibodies were all Alexa Fluor coupled (1:1000) and 

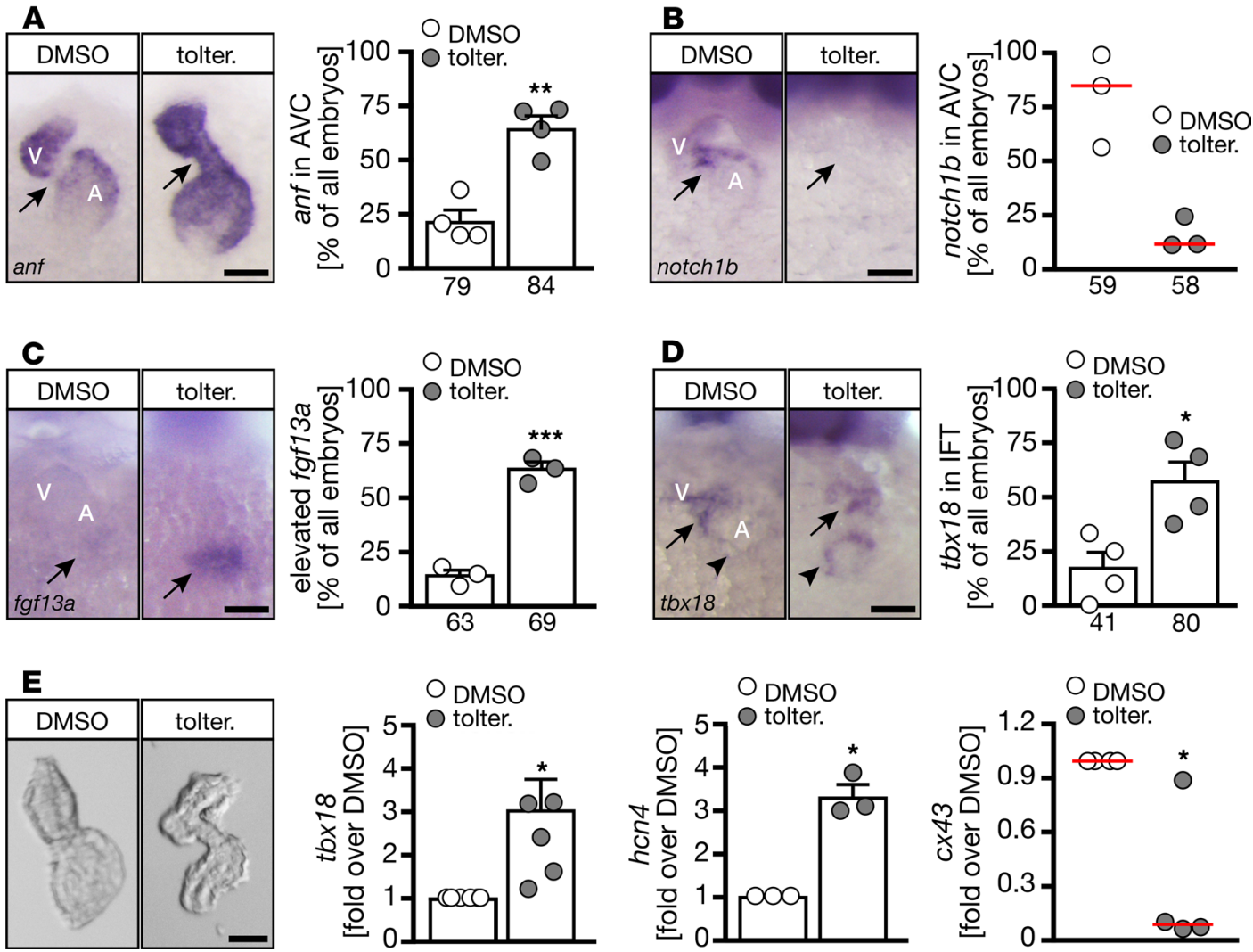

Figure 8. Tolterodine promotes pacemaker cell fate at the expense of AVC cells. (A-D) Representative images of whole-mount in situ hybridizations at $48 \mathrm{hpf}$. A, atrium; V, ventricle. Numbers of embryos studied are indicated below the bars. $n=3-4$ experiments. (A) AVC cells aberrantly express anf, which is characteristic of working myocardium and normally excluded from the AVC. ${ }^{*} P=0.0013,2$-tailed $t$ test with Welch's correction. (B) Upon tolterodine treatment, notch1b expression was lost. $P=0.100$, 2-tailed Mann-Whitney $U$ test. (C) Tolterodine provoked an increase in the pacemaker marker $f g f 13 a$. ${ }^{* * *} P=0.0005$, 2-tailed $t$ test with Welch's correction. (D) Tolterodine increased tbx18 expression in the area of the inflow tract. ${ }^{*} P=0.0158,2$-tailed $t$ test with Welch's correction. (E) Quantitative PCR ( by tolterodine. $n=3-6$ experiments. ${ }^{*} P=0.0142$ (hcn4), 0.0193 ( $c x 43$ ), and 0.0213 (tbx18), paired $t$ test. Scale bars: $50 \mu \mathrm{m}$. Bar graphs show mean \pm SEM (except for $\mathbf{B}$ and $t b x 18$ in $\mathbf{E}:$ red line is median). Circles show individual experiments. Arrows indicate changes in expression between panels.

included donkey anti-mouse IgG (H+L) Alexa Fluor 568 (Thermo Fisher Scientific, catalog A10037), anti-mouse IgG $(\mathrm{H}+\mathrm{L}), \mathrm{F}(\mathrm{ab}) 2$ Fragment (Alexa Fluor 488) (Cell Signaling Technology, catalog 4408S), and Goat anti-Rabbit IgG (H+L) Cross-Adsorbed Secondary Antibody, Alexa Fluor 568 (Thermo Fisher Scientific, catalog A-11011). The compound library was purchased from MilliporeSigma (LOPAC, catalog LO5100). Individual compounds were from Selleckchem (tolterodine tartrate) and Tocris (AF-DX 116, himbacine, solifenacin, telenzepine, zamifenacin).

Cloning and in vitro transcription of capped RNA. For sequence verification, the following plasmids were cloned: parts of the 5'-UTR along with the ORF of Chrm2a (ENSDART00000167233.1) were amplified by PCR using Phusion polymerase and cDNA of 24-hpf zebrafish embryos (forward: 5'-CTGCCCATAGCGATCTGAGT; reverse: 5'-TCGGGTGGAGCGAATGTT) and cloned via EcoRI/ XhoI into pCS2-GFP. To verify the sequence of Chrm3a, a fragment covering the upstream intron/ exon boundary of exon 3 of Chrm3a (ENSDART00000154251.1) was amplified by PCR using Phusion polymerase and cDNA of 24-hpf zebrafish embryos (forward: 5'-ACCCTTTAAATGTGCAAAGCA; reverse: 5'-TGTCCACCCAGAGGATCATAG) and cloned into pCR 4 by TOPO TA cloning (Life Technologies). For BRET assays, the ORFs (without stop codon) of Chrm2a and Chrm3a, respectively, were cloned into pcFLAG-Nluc via BspEI and XbaI.

Zebrafish and mouse lines. Adult zebrafish were generated in house to obtain fertilized eggs and maintained under standardized conditions in an automated housing system (ZebTEC, Tecniplast). Zebrafish lines used in this study were $\mathrm{AB}$ and EK WT lines generated in-house as well as previously described transgenic reporter lines to visualize cardiomyocytes or the nuclei of cardiomyocytes $(34,35)$. M2/M3 
A

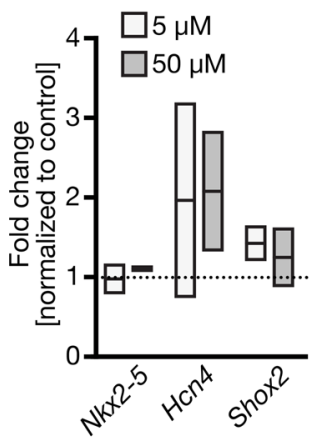

B

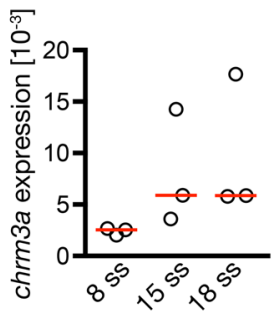

C

AVC elongation [ $>50 \%$ of all embryos]:

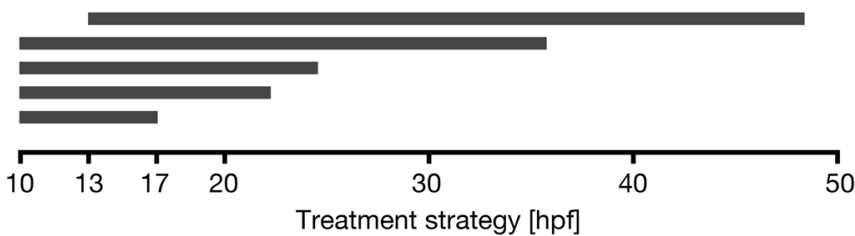

D
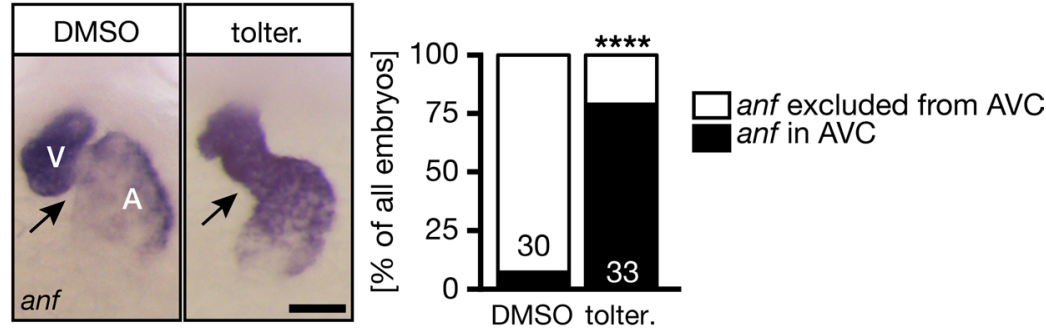

$\mathbf{E}$
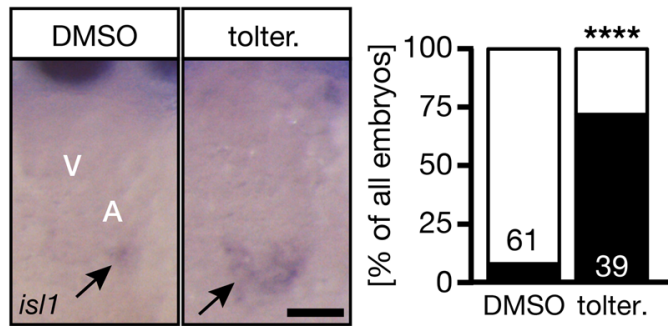

Figure 9. Treatment during CPC stages is sufficient to affect conduction system development. (A) Tolterodine treatment induced the expression of genes characteristic for pacemaker cells (Hcn4, Shox2) in cardiomyocytes differentiated from human iPSCs. Graph shows qPCR data of treatments. Line indicates mean; box shows minimum and maximum. (B) In zebrafish embryos, the muscarinic receptor M3a was increasingly expressed during CPC differentiation stages. qPCRs of zebrafish at the indicated stages. Chrm3a normalized to gapdh. Red line, median; ss, somite stages. (C) Schematic illustration of different treatment regimens in zebrafish embryos producing AVC elongation in more than $50 \%$ of the embryos. (D) Tolterodine treatment between tailbud and 20 ss is sufficient to induce anf expression in the presumptive AVC. Scale bar: $50 \mu \mathrm{m}$. Bar graph depicts the number of embryos with or without anf expression in the AVC. $n=2$ experiments. The number of individual embryos is given in the graph. ${ }^{* * *} P<0.0001,2$-sided Fisher's exact test. (E) Tolterodine treatment during heart progenitor specification and differentiation stages results in elevated is/1 expression that reaches into the atrium. Scale bar: $50 \mu \mathrm{m}$. Bar graph summarizes 2 experiments (embryo number indicated in the graph itself). ${ }^{* * *} P<0.0001,2$-sided Fisher's exact test. SAN, sinoatrial node.

double-KO mice were previously backcrossed to a C57BL/6N background. Clutches of fertilized zebrafish eggs were randomly split into different treatment groups. No sex determination was performed for zebrafish embryos. No embryos were excluded from experiments.

Zebrafish manipulations. For drug treatments, fertilized eggs were generated by natural matings and allowed to develop until tailbud stage at $28.5^{\circ} \mathrm{C}$. For the initial screen, embryos were transferred to 96-well plates (5 embryos/well; Nunc) and cultured in $300 \mu \mathrm{L}$ egg medium containing $1 \%$ DMSO or $50 \mu \mathrm{M}$ compound until the $48 \mathrm{hpf}$ stage was reached. Egg medium consisted of $1.5 \mathrm{~g}$ Instant Ocean (Happy Diskus), $0.375 \mathrm{~g}$ calcium sulfate (MilliporeSigma), and $0.18 \mathrm{mg}$ sodium hydrogen carbonate (MilliporeSigma) in $5 \mathrm{~L}$ demineralized water with a few drops of saturated methylene blue solution (MilliporeSigma). To prevent pigmentation, 0.003\% 1-phenyl-2-thiourea (MilliporeSigma) was added. For validation of individual hits, 10 embryos per well were immersed in a volume of $300 \mu \mathrm{L}$. All other treatments were done in 24-well plates in a total volume of $1 \mathrm{~mL}$. Embryos were assessed at $24 \mathrm{hpf}$ and $48 \mathrm{hpf}$ for overall morphology and cardiac contractions and subsequently fixed for further analysis. MOs were injected directly into the yolk at the 1-cell stage with an Eppendorf Femtojet equipped with a Narishige micromanipulator. All MOs were designed based on submitted sequences and produced by 
Gene Tools Inc. An MO targeting the translation start site with the sequence 5'-TCGCTCAGGTACAGCCACTCAGATC was used to knock down the muscarinic M2a receptor (Chrm2a MO). This receptor was previously targeted by another MO with the same results (21) as in this study. For the muscarinic $\mathrm{M} 3 \mathrm{a}$ receptor, an $\mathrm{MO}$ (Chrm3a MO) targeting the exon/intron boundary of the third exon was designed: 5'-CGGGCATATTCTGAAAAAGGAAAAT. Knockdown efficiency of the Chrm3a MO was tested by reverse transcription PCR (RT-PCR) (see below; full, uncut Western blot gels are available in the online supplemental material). In all injection experiments, noninjected controls were always included as well as embryos injected with a standard control MO, which did not target the zebrafish genome.

Analysis of cardiac performance in zebrafish. Individual zebrafish were placed under a whole-mount microscope and heart rates were counted. To minimize inconsistencies, the room temperature during counting was set to $26^{\circ} \mathrm{C}$. AV-blocks were calculated from atrial and ventricular heart rates. Embryos were considered to have a $50 \%$ AV-block when the ventricular heart rate was half the atrial heart rate (25\% AV-block: ventricular to atrial heart rate $=0.75 ; 75 \%$ AV-block: ventricular to atrial heart rate $=0.25)$. In addition, the occurrence of pericardiac and inflow tract edema was recorded.

Analysis of cardiac performance in Xenopus laevis. Xenopus embryos were treated from stage 12.5 until 42 in frog water containing $1 \%$ DMSO or $50 \mu \mathrm{M}$ tolterodine. Heart rates were counted manually and embryos were scored for arrhythmia and pericardiac edema.

Analysis of cardiac performance in mice. In vivo cardiac performance was assessed via transthoracic 2-dimensional echocardiography using the VisualSonics Vevo 2100 imaging system and a 30-MHz (MS400) transducer. Mice were anesthetized with $1.5 \%$ isoflurane via inhalation, and M-mode echocardiography was performed in the parasternal short-axis view at the level of the papillary muscle to assess several cardiac parameters, including left ventricular (LV) end-diastolic dimension and volume and LV fractional shortening and ejection fraction. Percentage of fractional shortening was calculated using the equation: ([LV internal dimension at diastole - LV internal dimension at systole]/LV internal dimension at systole) $\times 100 \%$. Percentage of ejection fraction was calculated using the equation: ([LV volume at diastole $-\mathrm{LV}$ volume; systole]/LV volume at diastole) $\times 100 \%$. All animal procedures and experiments were carried out according to the National Institutes of Health (NIH) Guide for the Care and Use of Laboratory Animals (National Academies Press, 2011) and approved by the Institutional Animal Care and Use Committee (IACUC) at Temple University.

BRET assays for $\beta$-arrestin 2 recruitment. Recruitment of $\beta$-arrestin 2 was measured in HEK293T cells (ATCC). Therefore, HEK cells were cultivated in DMEM supplemented with $4.5 \mathrm{~g} / \mathrm{L}$ glucose, $10 \%(v / v)$ fetal calf serum, $1000 \mathrm{U} / \mathrm{mL}$ penicillin, and $100 \mu \mathrm{g} / \mathrm{mL}$ streptomycin sulfate; were maintained at $37^{\circ} \mathrm{C}$ in a humidified $5 \% \mathrm{CO}_{2}$ atmosphere; and were routinely passaged. For assay preparation, $1 \times 10^{6}$ cells were seeded into a 55-mm culture dish and after 20 hours transiently transfected using the Effectene Transfection Reagent Kit (Qiagen) according to the manufacturer's instructions. The DNA of the receptor construct N-terminally modified with a FLAG tag and C-terminally fused to a nanoluciferase, a $\beta$-arrestin 2 construct $\mathrm{N}$-terminally modified with a HaloTag, and a GRK2 were simultaneously transfected in a ratio of 1:2:1. After 24 hours, cells were detached from the culture dish and counted, and 30,000 cells per well were reseeded into poly-D-lysineprecoated, white, 96-well cellGrade BRANDplates. During replating the HaloTag NanoBRET 618 ligand was added in accordance with the manufacturer's instructions (Promega). After 20 hours, BRET experiments were performed at $37^{\circ} \mathrm{C}$ using a Synergy Neo2 (BioTEK) plate reader equipped with a filter combination of $460 / 40$ $\mathrm{nm}$ and $620 / 20 \mathrm{~nm}$. Directly before the assay, cells were carefully washed twice with buffer $(140 \mathrm{mM} \mathrm{NaCl}$, $10 \mathrm{mM}$ HEPES, $5.4 \mathrm{mM} \mathrm{KCl}, 2 \mathrm{mM} \mathrm{CaCl}, 1 \mathrm{mM} \mathrm{MgCl}_{2}$ [pH 7.3]) and were afterward kept in $90 \mu \mathrm{L}$ buffer per well containing NanoGlo substrate (Promega). After a basal measurement was finished, $10 \mu \mathrm{L}$ of a ligand solution was added to each well, and a stimulated measurement was initiated. For data analysis, the BRET ratio was calculated by dividing acceptor signal by donor signal and afterward corrected for buffer conditions.

Immunofluorescence and whole-mount in situ hybridization. For immunofluorescence zebrafish embryos were fixed for 50 minutes with 1\% formaldehyde in PBS. After 1 hour of blocking in blocking solution (10\% normal goat serum [Vector Laboratories], $2 \mathrm{mg} / \mathrm{mL}$ BSA, and $0.2 \%$ saponine in PBS), embryos were incubated with primary antibodies diluted in blocking solution at $4^{\circ} \mathrm{C}$ overnight. Primary antibodies were detected using Alexa Fluor-coupled secondary antibodies. Stained embryos were mounted between 2 coverslips in Vectashield mounting medium (Vector Laboratories). Whole-mount in situ hybridization (WMISH) was done according to standard protocols $(36,37)$. The $h c n 4$, is 11 , notch $1 b$, and tbx $2 b$ plasmids were gifts from Jeroen Bakkers (Hubrecht Institute, Utrecht, the Netherlands), Ken Poss (Duke University, 
Durham, North Carolina, USA), and Gilbert Weidinger (Ulm University, Ulm, Germany). The $a m h c$, cspg2a, and $v m h$ c probes have been described before (38). For all other genes, PCR-amplified fragments according to GenBank sequences were cloned by TOPO TA cloning into pCRII, and digoxigenin-labeled in situ probes were transcribed after linearization of the plasmids. GenBank sequences were anf (GenBank NM_198800), fgf13a (GenBank MGC 101784), and tbx5a (GenBank NM_130915.1).

Treatment of human cardiomyocytes. Four-month-old cardiomyocytes were differentiated from WT human iPSCs as described before (39) and treated for 24 hours with either 5 or $50 \mu \mathrm{M}$ tolterodine dissolved in water. Three days after treatment, cells were collected and processed for qPCR analysis.

$R N A$ isolation and expression analysis. Total RNA was isolated from whole zebrafish embryos using the Quick-RNA Mini-Prep kit (Zymo Research) according to the manufacturer's instructions. cDNA was generated using Superscript II (Life Technologies) or the ProtoscriptII cDNA kit (NEB). Splice blocking by the Chr$\mathrm{m} 3 \mathrm{a}$ MO was analyzed by regular RT-PCR with primers amplifying a fragment of the third exon (forward: 5'-GACTCCAACAGCACCGAGTT; reverse: 5'-GAGATGACCCCAATGATGAGA). Gapdh was used as a control (forward: 5'-CCATCAACGGTCTTCTGTGTT; reverse: 5'-ACATTAAGTGGGGTGATGCAG) (all from Integrated DNA Technologies). For gene expression analysis in embryonic hearts, zebrafish hearts were isolated as described previously (40). Isolated hearts were lysed and cDNA was prepared using the TaqMan Gene Expression Cells-to-Ct kit from Ambion. qPCRs were run in duplicate or triplicate on a Roche Lightcycler 480 using the Roche Universal Probe System for detection and the following primers: Cx43 (forward: 5'-TCCACTCTTTACTTCTTTCCAGCTA; reverse: 5'-GAACAAGGATCCGGAAGATG; UP99), gapdh (forward: 5'-CAGGCATAATGGTTAAAGTTGGTA; reverse: 5'-CATGTAATCAAGGTCAATGAATGG; UP147), hon4 (forward: 5'-GCGCCTCATACGATACATCC; reverse: 5'-CTGGCCAGGTCATAGGTCAT; UP52), chrm3a (forward: 5'-AAAGAGCATCTTTTACCTTTCTGTG; reverse: 5'-AGGACATCAAACAGGTTCATACTG; UP106), and tbx18 (forward: 5'-CGGTGACTGCATACCAAAAC; reverse: 5'-CTTTTGCAAACGGATTCCTG; UP41). To analyze gene expression in cardiomyocyte cultures originating from iPSCs, RNA was isolated with the Agilent Absolutely RNA Microprep Kit, before it was transcribed into cDNA with Superscript II reverse transcriptase and analyzed by qPCR. Primers and universal probes for human genes were as follows (all from Integrated DNA Technologies): HCN4 (forward: 5'-GGTGTCCATCAACAACATGG; reverse: 5'-GCCTTGAAGAGCGCGTAG, UP38), NKX2-5 (forward: 5'-CCGGTTGGAACTGGGACT, reverse: 5'-GACGGCGAGATAGCAAAGG; UP81), and SHOX2 (forward: 5'-AACGTAGGTGCTTTAAGGATGC; reverse: 5'-GAAAGGACAAGGGCGTCAC; UP18).

Chrm3 expression in human BHM. Human BHM were analyzed to determine the time course of Chrm3 expression in a model of human heart development (41).

Microscopy. Live zebrafish embryos and those processed by WMISH were imaged using a Leica M125 whole-mount microscope equipped with a Leica IC80 HD camera. Fluorescent images were acquired either with a Leica M205FA microscope equipped with a DFC365FX camera or by confocal microscopy on a Leica SP5. Xenopus embryos were imaged using a UC50 camera on an SZX12 whole-mount microscope (both Olympus).

Sequence comparisons. Human and zebrafish muscarinic receptor sequences were compared using MultAlin (http://multalin.toulouse.inra.fr/multalin/) and protein-protein FASTA searches at https://fasta.bioch.virginia.edu/fasta_www2/fasta_list2.shtml with the following sequences: M2 (NP_000730.1), M3 (NP_000731.1), M2a (XP_005166056.1), and M3a (XP_021328763.1). Zebrafish sequences were sequence verified.

Statistics. All analyses were performed with the help of GraphPad Prism 5 and 6, respectively. Results were first assessed for normal distribution and subsequently analyzed using the tests as referred to in the figure legends. In general, a $P$ value less than 0.05 was considered statistically significant.

Study approval. All zebrafish husbandry and experiments described in here were approved by the local authorities at Ulm University (permit A.140). Mice were housed at Taconic Biosciences and analyzed at Temple University. All protocols to house and analyze mice were approved by the IACUC at Temple University (protocol ACUP 4602).

\section{Author contributions}

MSB, MDB, M Kauk, TCT, LAG, SM, CD, M Kustermann, MR, YC, FR, SL, M Kühl, DGT, and MP performed experiments. MSB, MDB, M Kauk, TCT, LAG, SM, CD, M Kustermann, MR, FR, SL, WHZ, M Kühl, CH, DGT, and MP analyzed data. AM, M Kauk, CH, WHZ, M Kühl, DGT, and MP designed experiments. MP conceived the study. WHZ, JW, DGT, and MP wrote the manuscript. 


\section{Acknowledgments}

We thank Sandra Burczyk, Sophia Aicher, and Carina Riedmüller for excellent fish care and the lab of Antje Gohla for help with initial mouse experiments.. This work was supported by the Boehringer Ingelheim Ulm University BioCenter (C9, to MP), NIH grant HL105414 (to DGT), and American Heart Association grant 17SDG33400114 (to LAG). TCT and MSB were scholars of the International Graduate School in Molecular Medicine at Ulm University (funded by the German Excellence Initiative of the DFG). The lab of MP is further supported by grants from the DFG (PH144/4-1 and PH144/6-1). JW was funded by the Intramural Research Program of the National Institute of Diabetes and Digestive and Kidney Diseases (Bethesda, Maryland, USA). WHZ is supported by the DZHK, the German Federal Ministry for Science and Education (BMBF FKZ 13GW0007A [CIRM-ET3]), the German Research Foundation (DFG ZI 708/10-1; SFB 937 TP18, SFB 1002 TPs C04, S1; IRTG 1816 RP12), the Foundation Leducq, and the NIH (U01HL099997).

Address correspondence to: Melanie Philipp, Department of Experimental and Clinical Pharmacology and Pharmacogenomics, Division of Pharmacogenomics, University of Tuebingen, Wilhelmstrasse 56, 72074 Tuebingen, Germany. Phone: 49.7071.29.72271; Email: melanie.philipp@uni-tuebingen.de.

LAG's present address is: College of Veterinary Medicine, University of Missouri, Columbia, Missouri, USA.

1. John RM, Kumar S. Sinus node and atrial arrhythmias. Circulation. 2016;133(19):1892-1900.

2. Hu YF, Dawkins JF, Cho HC, Marbán E, Cingolani E. Biological pacemaker created by minimally invasive somatic reprogramming in pigs with complete heart block. Sci Transl Med. 2014;6(245):245ra94.

3. Husse B, Franz WM. Generation of cardiac pacemaker cells by programming and differentiation. Biochim Biophys Acta. 2016;1863(7 Pt B):1948-1952.

4. Brand T. Tbx18 and the generation of a biological pacemaker. Are we there yet? J Mol Cell Cardiol. 2016;97:263-265.

5. Donahue JK, et al. Focal modification of electrical conduction in the heart by viral gene transfer. Nat Med. 2000;6(12):1395-1398

6. Keßler M, Rottbauer W, Just S. Recent progress in the use of zebrafish for novel cardiac drug discovery. Expert Opin Drug Discov. 2015;10(11):1231-1241.

7. Poon KL, Liebling M, Kondrychyn I, Brand T, Korzh V. Development of the cardiac conduction system in zebrafish. Gene Expr Patterns. 2016;21(2):89-96.

8. de Pater E, et al. Distinct phases of cardiomyocyte differentiation regulate growth of the zebrafish heart. Development. 2009;136(10):1633-1641.

9. Hoffmann S, et al. Islet1 is a direct transcriptional target of the homeodomain transcription factor Shox 2 and rescues the Shox2-mediated bradycardia. Basic Res Cardiol. 2013;108(2):339.

10. Peal DS, Lynch SN, Milan DJ. Patterning and development of the atrioventricular canal in zebrafish. J Cardiovasc Transl Res. 2011;4(6):720-726.

11. Griffin A, Hamling KR, Knupp K, Hong S, Lee LP, Baraban SC. Clemizole and modulators of serotonin signalling suppress seizures in Dravet syndrome. Brain. 2017;140(3):669-683.

12. Wang $G$, et al. First quantitative high-throughput screen in zebrafish identifies novel pathways for increasing pancreatic $\beta$-cell mass. Elife. 2015;4:e08261.

13. Stainier DY. Zebrafish genetics and vertebrate heart formation. Nat Rev Genet. 2001;2(1):39-48.

14. Chapple CR. Muscarinic receptor antagonists in the treatment of overactive bladder. Urology. 2000;55(Suppl 5A):33-46.

15. Wang Z, Shi H, Wang H. Functional M3 muscarinic acetylcholine receptors in mammalian hearts. Br J Pharmacol. 2004;142(3):395-408.

16. Gomeza J, et al. Pronounced pharmacologic deficits in M2 muscarinic acetylcholine receptor knockout mice. Proc Natl Acad Sci US A. 1999;96(4):1692-1697.

17. Yamada M, et al. Mice lacking the M3 muscarinic acetylcholine receptor are hypophagic and lean. Nature. 2001;410(6825):207-212.

18. Benes J, Varejkova E, Farar V, Novakova M, Myslivecek J. Decrease in heart adrenoceptor gene expression and receptor number as compensatory tool for preserved heart function and biological rhythm in M(2) KO animals. Naunyn Schmiedebergs Arch Pharmacol. 2012;385(12):1161-1173.

19. Fisher JT, Vincent SG, Gomeza J, Yamada M, Wess J. Loss of vagally mediated bradycardia and bronchoconstriction in mice lacking M2 or M3 muscarinic acetylcholine receptors. FASEB J. 2004;18(6):711-713.

20. Machleidt T, et al. NanoBRET - a novel BRET platform for the analysis of protein-protein interactions. ACS Chem Biol. 2015;10(8):1797-1804.

21. Hsieh DJ, Liao CF. Zebrafish M2 muscarinic acetylcholine receptor: cloning, pharmacological characterization, expression patterns and roles in embryonic bradycardia. Br J Pharmacol. 2002;137(6):782-792.

22. Wallis RM. Pre-clinical and clinical pharmacology of selective muscarinic M3 receptor antagonists. Life Sci. 1995;56(11-12):861-868.

23. Dietrich AC, Lombardo VA, Veerkamp J, Priller F, Abdelilah-Seyfried S. Blood flow and Bmp signaling control endocardial chamber morphogenesis. Dev Cell. 2014;30(4):367-377.

24. Christoffels VM, Smits GJ, Kispert A, Moorman AF. Development of the pacemaker tissues of the heart. Circ Res. 2010;106(2):240-254.

25. Pandur P, Sirbu IO, Kühl SJ, Philipp M, Kühl M. Islet1-expressing cardiac progenitor cells: a comparison across species. Dev Genes Evol. 2013;223(1-2):117-129. 
26. Bressan M, Liu G, Mikawa T. Early mesodermal cues assign avian cardiac pacemaker fate potential in a tertiary heart field. Science. 2013;340(6133):744-748.

27. Christoffels VM, et al. Formation of the venous pole of the heart from an Nkx2-5-negative precursor population requires Tbx18 Circ Res. 2006;98(12):1555-1563.

28. Kelder TP, et al. The sinus venosus myocardium contributes to the atrioventricular canal: potential role during atrioventricular node development? J Cell Mol Med. 2015;19(6):1375-1389.

29. Fregoso SP, Hoover DB. Development of cardiac parasympathetic neurons, glial cells, and regional cholinergic innervation of the mouse heart. Neuroscience. 2012;221:28-36.

30. Hildreth V, Webb S, Bradshaw L, Brown NA, Anderson RH, Henderson DJ. Cells migrating from the neural crest contribute to the innervation of the venous pole of the heart. J Anat. 2008;212(1):1-11.

31. Kapoor N, Liang W, Marbán E, Cho HC. Direct conversion of quiescent cardiomyocytes to pacemaker cells by expression of Tbx18. Nat Biotechnol. 2013;31(1):54-62.

32. Greulich F, Trowe MO, Leffler A, Stoetzer C, Farin HF, Kispert A. Misexpression of Tbx18 in cardiac chambers of fetal mice interferes with chamber-specific developmental programs but does not induce a pacemaker-like gene signature. J Mol Cell Cardiol. 2016;97:140-149.

33. Austin KM, et al. Molecular mechanisms of arrhythmogenic cardiomyopathy. Nat Rev Cardiol. 2019;16(9):519-537.

34. Huang CJ, Tu CT, Hsiao CD, Hsieh FJ, Tsai HJ. Germ-line transmission of a myocardium-specific GFP transgene reveals critical regulatory elements in the cardiac myosin light chain 2 promoter of zebrafish. Dev Dyn. 2003;228(1):30-40.

35. Mably JD, Mohideen MA, Burns CG, Chen JN, Fishman MC. heart of glass regulates the concentric growth of the heart in zebrafish. Curr Biol. 2003;13(24):2138-2147.

36. Moens C. Whole mount RNA in situ hybridization on zebrafish embryos: hybridization. CSH Protoc. 2008;2008:pdb.prot5037.

37. Thisse C, Thisse B. High-resolution in situ hybridization to whole-mount zebrafish embryos. Nat Protoc. 2008;3(1):59-69.

38. Burkhalter MD, Fralish GB, Premont RT, Caron MG, Philipp M. Grk51 controls heart development by limiting mTOR signaling during symmetry breaking. Cell Rep. 2013;4(4):625-632.

39. Burridge PW, et al. Chemically defined generation of human cardiomyocytes. Nat Methods. 2014;11(8):855-860

40. Burns CG, MacRae CA. Purification of hearts from zebrafish embryos. BioTechniques. 2006;40(3):274.

41. Zeidler S, et al. Computational detection of stage-specific transcription factor clusters during heart development. Front Genet. $2016 ; 7: 33$ 